\title{
LINEAR COMBINATIONS OF HARMONIC QUASICONFORMAL MAPPINGS CONVEX IN ONE DIRECTION
}

\author{
Yong Sun, Antti Rasila and Yue-Ping Jiang
}

\begin{abstract}
In this paper, we introduce a new class $\mathscr{S}_{H}(k, \gamma ; \phi)$ of harmonic quasiconformal mappings, where $k \in[0,1), \gamma \in[0, \pi)$ and $\phi$ is an analytic function. Sufficient conditions for the linear combinations of mappings in such classes to be in a similar class, and convex in a given direction, are established. In particular, we prove that the images of linear combinations in this class, for special choices of $\gamma$ and $\phi$, are convex.
\end{abstract}

\section{Introduction}

A complex-valued function $f$ defined in the open unit disk $\mathbf{D}=\{z \in \mathbf{C}$ : $|z|<1\}$ is called harmonic if $f$ is twice continuously differentiable and satisfies $\Delta f=4 f_{z \bar{z}}=0$. Let $\mathscr{H}$ denote the class of all complex-valued harmonic functions $f$ in $\mathbf{D}$ normalized by the condition $f(0)=f_{z}(0)-1=0$. Let $\mathscr{S}_{H}$ be the subclass of $\mathscr{H}$ consisting of univalent and sense-preserving functions. Such functions can be written in the form $f=h+\bar{g}$, where

$$
h(z)=z+\sum_{n=2}^{\infty} a_{n} z^{n} \quad \text { and } \quad g(z)=\sum_{n=1}^{\infty} b_{n} z^{n}
$$

are analytic in $\mathbf{D}$ and the Jacobian $J_{f}(z)=\left|h^{\prime}(z)\right|^{2}-\left|g^{\prime}(z)\right|^{2}>0$, or equivalently, the analytic complex dilatation $\omega=g^{\prime} / h^{\prime}$ of $f$ satisfies $|\omega|<1$ in D. The classical class $\mathscr{S}$ of analytic univalent and normalized functions in $\mathbf{D}$ is a subclass of $\mathscr{S}_{H}$ with $g(z) \equiv 0$. The class of all functions $f \in \mathscr{S}_{H}$ with the additional property that $f_{\bar{z}}(0)=0$ is denoted by $\mathscr{S}_{H}^{0}$. We refer to $[6,9,10]$ for the basic theory of harmonic mappings, and $[2,3,5,14,16,23]$ for some recent investigations on the topic.

If a univalent harmonic mapping $f=h+\bar{g}$ satisfies the condition

$$
\left|\frac{g^{\prime}(z)}{h^{\prime}(z)}\right| \leq k<1 \quad(z \in \mathbf{D})
$$

2010 Mathematics Subject Classification. Primary: 30C62; Secondary: 30C45.

Key words and phrases. Univalent harmonic mapping; harmonic $K$-quasiconformal mapping; linear combination; convex in one direction.

Received July 16, 2015; revised October 13, 2015. 
then $f$ is called a harmonic $K$-quasiconformal mapping in $\mathbf{D}$, where $K=\frac{1+k}{1-k}$. Let $\mathscr{S}_{H}(k)$ be the subclass of $\mathscr{S}_{H}^{0}$ consisting of harmonic $K$-quasiconformal mappings. Recently, several authors derived the conditions for univalent harmonic mappings to be $K$-quasiconformal, see (for example) the works $[1,11,12$, $18]$ and the references therein.

A domain $\Omega \subset \mathbf{C}$ is said to be convex in the direction $\gamma \in[0, \pi)$, if for all $a \in \mathbf{C}$, the set $\Omega \cap\left\{a+t e^{i \gamma}: t \in \mathbf{R}\right\}$ is either connected or empty. In particular, a domain is convex in the direction of the real (imaginary) axis if every line parallel to the real (imaginary) axis has either an empty intersection or a connected intersection with the domain. A function is said to be convex in the direction $\gamma$ if it maps $\mathbf{D}$ univalently onto a domain convex in the direction $\gamma$.

Let $f_{1}=h_{1}+\overline{g_{1}}$ and $f_{2}=h_{2}+\overline{g_{2}}$ be two univalent harmonic mappings in D with respective dilatations $\omega_{1}$ and $\omega_{2}$. Then, the linear combination $f$ of $f_{1}$ and $f_{2}$ is given by

$$
\begin{aligned}
f & =t f_{1}+(1-t) f_{2}=\left[t h_{1}+(1-t) h_{2}\right]+\left[t \overline{g_{1}}+(1-t) \overline{g_{2}}\right] \\
& =h+\bar{g}, \quad(0 \leq t \leq 1) .
\end{aligned}
$$

Even if $f$ and $g$ are convex analytic functions, Macgregor [15] has shown that $t f+(1-t) g(0 \leq t \leq 1)$ need not be univalent. For results on the analytic linear combination, see (for example) [4, 21]. For linear combinations of harmonic functions, Dorff and Rolf [8] provided sufficient conditions for the linear combination $f=t f_{1}+(1-t) f_{2}$ to be univalent and convex in the direction of the imaginary axis under the assumption that $\omega_{1}=\omega_{2}$. Furthermore, Wang et al.

[22] proved that the linear combination $f=t f_{1}+(1-t) f_{2}$ with $h_{j}+g_{j}=\frac{z}{1-z}$ $(j=1,2)$ is univalent and convex in the direction of the real axis. Recently, Kumar et al. [13] established that the linear combination $f=t f_{1}+(1-t) f_{2}$ with $h_{j}+g_{j}=\frac{z\left(1-\alpha_{j} z\right)}{1-z^{2}}\left(-1 \leq \alpha_{j} \leq 1 ; j=1,2\right)$ is univalent and convex in the direction of the imaginary axis.

Let $\mathscr{A}$ be the subclass of $\mathscr{S}_{H}^{0}$ consisting of analytic functions. For $k \in[0,1)$, $\gamma \in[0, \pi)$ and $\phi \in \mathscr{A}$, consider the following subclass $\mathscr{S}_{H}(k, \gamma ; \phi)$ of $\mathscr{S}_{H}$ defined by

$$
\mathscr{S}_{H}(k, \gamma ; \phi):=\left\{f=h+\bar{g} \in \mathscr{S}_{H}(k): h-e^{2 i \gamma} g=\phi\right\} .
$$

For simplicity, we write $\mathscr{S}_{H}(k, 0 ; \phi)=: \mathscr{S}_{H}^{-}(k ; \phi)$ and $\mathscr{S}_{H}\left(k, \frac{\pi}{2} ; \phi\right)=: \mathscr{S}_{H}^{+}(k ; \phi)$. These subclasses of harmonic mappings were introduced in $[17,24]$ for specific choices of $\gamma$ and $\phi$.

In this paper, we derive sufficient conditions for the linear combinations of harmonic quasiconformal mappings to be univalent and convex in a given direction. In particular, we prove that the images of linear combinations in this subclass, for special choices of $\gamma$ and $\phi$, are convex. 


\section{Preliminary results}

The proofs of our main results are based on the following lemmas.

Lemma 1 (see [6]). A sense-preserving harmonic function $f=h+\bar{g}$ in $\mathbf{D}$ is a univalent mapping of $\mathbf{D}$ onto a domain convex in the direction of the real (resp. imaginary) axis if and only if $h-g$ (resp. $h+g)$ is an analytic univalent mapping of $\mathbf{D}$ onto a domain convex in the direction of the real (resp. imaginary) axis.

It is clear that Lemma 1 of Clunie and Sheil-Small can easily be generalized to a domain convex in the direction $\gamma$.

Lemma 2. A sense-preserving harmonic function $f=h+\bar{g}$ in $\mathbf{D}$ is a univalent mapping of $\mathbf{D}$ onto a domain convex in the direction $\gamma$ if and only if $h-e^{2 i \gamma} g$ is an analytic univalent mapping of $\mathbf{D}$ onto a domain convex in the direction $\gamma$.

Lemma 3 (see [19]). Let $f$ be an analytic function in $\mathbf{D}$ with $f(0)=0$ and $f^{\prime}(0) \neq 0$ and let

$$
\kappa(z)=\frac{z}{\left(1+z e^{i \theta}\right)\left(1+z e^{-i \theta}\right)} \quad(\theta \in \mathbf{R})
$$

If

$$
\Re\left(\frac{z f^{\prime}(z)}{\kappa(z)}\right)>0 \quad(z \in \mathbf{D}),
$$

then $f$ is convex in the direction of the real axis.

Lemma 4 (see [20]). Let $\varphi(z)$ be a non-constant function regular in $\mathbf{D}$. The function $\varphi(z)$ maps $\mathbf{D}$ univalently onto a domain convex in the direction of imaginary axis, if and only if there are numbers $\mu$ and $v, 0 \leq \mu<2 \pi$ and $0 \leq v \leq \pi$ such that

$$
\Re\left(-i e^{i \mu}\left(1-2 z e^{-i \mu} \cos v+z^{2} e^{-2 i \mu}\right) \varphi^{\prime}(z)\right) \geq 0 \quad(z \in \mathbf{D}) .
$$

Lemma 5. If $f_{j} \in \mathscr{S}_{H}(k, \gamma ; \phi)(j=1,2)$, then the dilatation $\omega$ of the linear combination $f=t f_{1}+(1-t) f_{2}(0 \leq t \leq 1)$ satisfies

$$
|\omega|=\left|\frac{t g_{1}^{\prime}+(1-t) g_{2}^{\prime}}{t h_{1}^{\prime}+(1-t) h_{2}^{\prime}}\right| \leq k<1 .
$$

Proof. Since $h_{j}-e^{2 i \gamma} g_{j}=\phi$ and $g_{j}^{\prime}=\omega_{j} h_{j}^{\prime}(j=1,2)$, we get

$$
h_{j}^{\prime}=\frac{\phi^{\prime}}{1-e^{2 i \gamma} \omega_{j}} \quad(j=1,2) .
$$

We obtain a new harmonic mapping as follows

$$
f=t f_{1}+(1-t) f_{2}=\left[t h_{1}+(1-t) h_{2}\right]+\overline{\left[t g_{1}+(1-t) g_{2}\right]}=h+\bar{g},
$$


and the dilatation $\omega=g^{\prime} / h^{\prime}$ satisfies the condition

$$
\begin{aligned}
|\omega| & =\left|\frac{t g_{1}^{\prime}+(1-t) g_{2}^{\prime}}{t h_{1}^{\prime}+(1-t) h_{2}^{\prime}}\right|=\left|\frac{\frac{t \omega_{1} \phi^{\prime}}{1-e^{2 i \gamma} \omega_{1}}+\frac{(1-t) \omega_{2} \phi^{\prime}}{1-e^{2 i \gamma} \omega_{2}}}{\frac{t \phi^{\prime}}{1-e^{2 i \gamma} \omega_{1}}+\frac{(1-t) \phi^{\prime}}{1-e^{2 i \gamma} \omega_{2}}}\right| \\
& =\left|\frac{\frac{t \omega_{1}}{1-e^{2 i \gamma} \omega_{1}}+\frac{(1-t) \omega_{2}}{1-e^{2 i \gamma} \omega_{2}}}{\frac{t}{1-e^{2 i \gamma} \omega_{1}}+\frac{1-t}{1-e^{2 i \gamma} \omega_{2}}}\right| .
\end{aligned}
$$

From (5) it follows that $|\omega| \leq k$ if and only if

$$
k^{2}\left|\frac{t}{1-e^{2 i \gamma} \omega_{1}}+\frac{1-t}{1-e^{2 i \gamma} \omega_{2}}\right|^{2}-\left|\frac{t \omega_{1}}{1-e^{2 i \gamma} \omega_{1}}+\frac{(1-t) \omega_{2}}{1-e^{2 i \gamma} \omega_{2}}\right|^{2} \geq 0 .
$$

Let

$$
\omega_{j}=\rho_{j} e^{i \theta_{j}} \quad\left(0 \leq \rho_{j} \leq k<1, \theta_{j} \in \mathbf{R} ; j=1,2\right)
$$

and

$$
\Phi:=\frac{2 t(1-t)}{\left|1-e^{2 i \gamma} \omega_{1}\right|^{2}\left|1-e^{2 i \gamma} \omega_{2}\right|^{2}} \geq 0
$$

Then we have

$$
\begin{aligned}
& k^{2}\left|\frac{t}{1-e^{2 i \gamma} \omega_{1}}+\frac{1-t}{1-e^{2 i \gamma} \omega_{2}}\right|^{2}-\left|\frac{t \omega_{1}}{1-e^{2 i \gamma} \omega_{1}}+\frac{(1-t) \omega_{2}}{1-e^{2 i \gamma} \omega_{2}}\right|^{2} \\
& =\frac{t^{2}\left(k^{2}-\left|\omega_{1}\right|^{2}\right)}{\left|1-e^{2 i \gamma} \omega_{1}\right|^{2}}+\frac{(1-t)^{2}\left(k^{2}-\left|\omega_{2}\right|^{2}\right)}{\left|1-e^{2 i \gamma} \omega_{2}\right|^{2}} \\
& +2 t(1-t) \Re\left(\frac{k^{2}-\omega_{1} \overline{\omega_{2}}}{\left(1-e^{2 i \gamma} \omega_{1}\right)\left(1-e^{-2 i \gamma} \overline{\omega_{2}}\right)}\right) \\
& \geq \frac{2 t(1-t)}{\left|1-e^{2 i \gamma} \omega_{1}\right|^{2}\left|1-e^{2 i \gamma} \omega_{2}\right|^{2}} \Re\left(\left(k^{2}-\omega_{1} \overline{\omega_{2}}\right)\left(1-e^{-2 i \gamma} \overline{\omega_{1}}\right)\left(1-e^{2 i \gamma} \omega_{2}\right)\right) \\
& =\Phi\left(\left(k^{2}-\rho_{1}^{2} \rho_{2}^{2}\right)+\rho_{1}\left(\rho_{2}^{2}-k^{2}\right) \cos \left(2 \gamma+\theta_{1}\right)\right. \\
& \left.+\rho_{2}\left(\rho_{1}^{2}-k^{2}\right) \cos \left(2 \gamma+\theta_{2}\right)+\rho_{1} \rho_{2}\left(k^{2}-1\right) \cos \left(\theta_{2}-\theta_{1}\right)\right) \\
& \geq \Phi\left(\left(k^{2}-\rho_{1}^{2} \rho_{2}^{2}\right)-\rho_{1}\left(k^{2}-\rho_{2}^{2}\right)-\rho_{2}\left(k^{2}-\rho_{1}^{2}\right)-\rho_{1} \rho_{2}\left(1-k^{2}\right)\right) \\
& =\Phi\left(k^{2}-\rho_{1} \rho_{2}\right)\left(1-\rho_{1}\right)\left(1-\rho_{2}\right) \geq 0 \text {. }
\end{aligned}
$$

The proof of Lemma 5 is thus completed. 
Obviously, we may generalize Lemma 5 as follows.

Lemma 6. If $f_{j} \in \mathscr{S}_{H}(k, \gamma ; \phi)(j=1,2, \ldots, n)$, then the dilatation $\omega$ of the linear combination $f=t_{1} f_{1}+t_{2} f_{2}+\cdots+t_{n} f_{n}$ satisfies

$$
|\omega|=\left|\frac{t_{1} g_{1}^{\prime}+t_{2} g_{2}^{\prime}+\cdots+t_{n} g_{n}^{\prime}}{t_{1} h_{1}^{\prime}+t_{2} h_{2}^{\prime}+\cdots+t_{n} h_{n}^{\prime}}\right| \leq k<1,
$$

where $0 \leq t_{j} \leq 1$ and $t_{1}+t_{2}+\cdots+t_{n}=1$.

\section{Main results}

We begin by presenting sufficient conditions for the linear combinations for the class $\mathscr{S}_{H}(k, \gamma ; \phi)$ to preserve certain properties of mappings.

THEOREM 1. Let $f_{j}=h_{j}+\overline{g_{j}} \in \mathscr{S}_{H}(k, \gamma ; \phi)(j=1,2)$. If $\phi$ is convex in the direction $\gamma$, then $f=t f_{1}+(1-t) f_{2} \in \mathscr{S}_{H}(k, \gamma ; \phi)(0 \leq t \leq 1)$, and it is convex in the direction $\gamma$.

Proof. In view of Lemma 5, we know that the dilatation $\omega$ of $f=t f_{1}+$ $(1-t) f_{2}$ satisfies $|\omega| \leq k$. Since $h_{j}-e^{2 i \gamma} g_{j}=\phi(j=1,2)$, we have

$$
\begin{aligned}
h-e^{2 i \gamma} g & =\left[t h_{1}+(1-t) h_{2}\right]-e^{2 i \gamma}\left[t g_{1}+(1-t) g_{2}\right] \\
& =t\left(h_{1}-e^{2 i \gamma} g_{1}\right)+(1-t)\left(h_{2}-e^{2 i \gamma} g_{2}\right)=\phi,
\end{aligned}
$$

which is convex in the direction $\gamma$ by the assumption. Thus, from Lemma 2, we see that $f \in \mathscr{S}_{H}(k, \gamma ; \phi)$ and convex in the direction $\gamma$.

In view of Theorem 1 and Lemma 6 , we have the following result.

Corollary 1. Let $f_{j}=h_{j}+\overline{g_{j}} \in \mathscr{S}_{H}(k, \gamma ; \phi)(j=1,2, \ldots, n)$. If $\phi$ is convex in the direction $\gamma$, then $f=\sum_{j=1}^{n} t_{j} f_{j} \in \mathscr{S}_{H}(k, \gamma ; \phi)\left(0 \leq t_{j} \leq 1, \sum_{j=1}^{n} t_{j}=1\right)$, and it is convex in the direction $\gamma$.

Remark 1. If we set $n=2, \gamma=0$ and $\phi=\frac{z}{1-z}$ in Corollary 1 , then it reduces to the result of Wang et al. [22, Theorem 3].

By making use of Theorem 1, we can obtain some interesting results for specific choices of $\gamma$ and $\phi$.

COROLlaRY 2. Let $f_{j}=h_{j}+\overline{g_{j}} \in \mathscr{S}_{H}(k, \gamma ; \phi)(j=1,2)$, where

$$
\phi(z)=\int_{0}^{z} \frac{e^{i \gamma} d \zeta}{\left(1+\zeta e^{i \theta}\right)\left(1+\zeta e^{-i \theta}\right)} \quad(\theta \in \mathbf{R}) .
$$

Then $f=t f_{1}+(1-t) f_{2} \in \mathscr{S}_{H}(k, \gamma ; \phi)(0 \leq t \leq 1)$, and it is convex in the direction $\gamma$. 
Proof. By setting $\kappa(z)$ by (3), we find that

$$
\begin{aligned}
\Re\left(\frac{z e^{-i \gamma}\left(h^{\prime}-e^{2 i \gamma} g^{\prime}\right)}{\kappa(z)}\right) & =\Re\left(\frac{z e^{-i \gamma}}{\kappa(z)}\left[t\left(h_{1}^{\prime}-e^{2 i \gamma} g_{1}^{\prime}\right)+(1-t)\left(h_{2}^{\prime}-e^{2 i \gamma} g_{2}^{\prime}\right)\right]\right) \\
& =t \cdot \Re\left(\frac{z e^{-i \gamma} \phi^{\prime}(z)}{\kappa(z)}\right)+(1-t) \cdot \Re\left(\frac{z e^{-i \gamma} \phi^{\prime}(z)}{\kappa(z)}\right) \\
& =t+(1-t)=1>0 .
\end{aligned}
$$

Therefore, by Lemma 3, we see that $e^{-i \gamma}\left(h-e^{2 i \gamma} g\right)$ is convex in the direction of the real axis, and hence the function $h-e^{2 i \gamma} g$ is convex in the direction $\gamma$. Furthermore, by Lemma 2 and Lemma 5, we deduce that $f \in \mathscr{S}_{H}(k, \gamma ; \phi)$ and convex in the direction $\gamma$.

Corollary 3. Suppose that $\alpha \in[-1,1], \theta \in(0, \pi)$ and $A, B \geq 0, A+B \neq 0$. Let $f_{j}=h_{j}+\overline{g_{j}} \in \mathscr{S}_{H}^{+}(k ; \phi) \quad(j=1,2)$, where

$$
\phi=A \cdot \frac{z(1-\alpha z)}{1-z^{2}}+B \cdot \frac{1}{2 i \sin \theta} \log \left(\frac{1+z e^{i \theta}}{1+z e^{-i \theta}}\right),
$$

then $f=t f_{1}+(1-t) f_{2} \in \mathscr{S}_{H}^{+}(k ; \phi)(0 \leq t \leq 1)$, and it is convex in the direction of the imaginary axis.

Proof. By taking $\mu=v=\frac{\pi}{2}$ in (4), we find that

$$
\begin{aligned}
\Re\left(\left(1-z^{2}\right) \phi^{\prime}(z)\right)= & A \cdot \Re\left(\frac{1-2 \alpha z+z^{2}}{1-z^{2}}\right)+B \cdot \Re\left(\frac{1-z^{2}}{\left(1+z e^{i \theta}\right)\left(1+z e^{-i \theta}\right)}\right) \\
= & A \cdot \frac{\left(1-|z|^{2}\right)\left(1-2 \alpha \Re(z)+|z|^{2}\right)}{\left|1-z^{2}\right|^{2}} \\
& +B \cdot \frac{\left(1-|z|^{2}\right)\left(1+2 \cos \theta \Re(z)+|z|^{2}\right)}{\left|1+z e^{i \theta}\right|^{2} \cdot\left|1+z e^{-i \theta}\right|^{2}}>0 .
\end{aligned}
$$

Therefore, by Lemma $4, \phi$ is convex in the direction of the imaginary axis, and hence by Theorem 1 with $\gamma=\frac{\pi}{2}$, we see that $f \in \mathscr{S}_{H}^{+}(k ; \phi)$ and $f$ is convex in the direction of the imaginary axis.

Remark 2. The main results of Kumar et al. [13] reduce to special cases of Corollary 3.

Since the function defined by (8) is convex in the direction of the real axis (see [7]), we can obtain the following result. 
Corollary 4. Suppose that $A, B \geq 0, A+B \neq 0$ and $c \in[-2,2]$. Let $f_{j}=$ $h_{j}+\overline{g_{j}} \in \mathscr{S}_{H}^{-}(k ; \phi)(j=1,2)$, where

$$
\phi=A \cdot \log \left(\frac{1+z}{1-z}\right)+B \cdot \frac{z}{1+c z+z^{2}},
$$

then $f=t f_{1}+(1-t) f_{2} \in \mathscr{S}_{H}^{-}(k ; \phi)(0 \leq t \leq 1)$, and it is convex in the direction of the real axis.

Theorem 2. Let $f_{1}=h_{1}+\overline{g_{1}} \in \mathscr{S}_{H}(k, \gamma ; \phi)$ and $f_{2}=h_{2}+\overline{g_{2}} \in \mathscr{S}_{H}(k, \gamma ; \psi)$. Suppose that

$$
\Re\left(k^{2} h_{1}^{\prime} \overline{h_{2}^{\prime}}-g_{1}^{\prime} \overline{g_{2}^{\prime}}\right) \geq 0
$$

and $t \phi+(1-t) \psi$ is convex in the direction $\gamma$, then $f=t f_{1}+(1-t) f_{2} \in \mathscr{S}_{H}(k)$ $(0 \leq t \leq 1)$, and it is convex in the direction $\gamma$.

Proof. For $g_{j}^{\prime}=\omega_{j} h_{j}^{\prime}$ satisfy the conditions $\left|\omega_{j}\right| \leq k<1(j=1,2)$, we have

$$
|\omega|=\left|\frac{t g_{1}^{\prime}+(1-t) g_{2}^{\prime}}{t h_{1}^{\prime}+(1-t) h_{2}^{\prime}}\right|=\left|\frac{t \omega_{1} h_{1}^{\prime}+(1-t) \omega_{2} h_{2}^{\prime}}{t h_{1}^{\prime}+(1-t) h_{2}^{\prime}}\right| .
$$

By assumption, it follows that

$$
\begin{aligned}
k^{2} \mid t h_{1}^{\prime} & +\left.(1-t) h_{2}^{\prime}\right|^{2}-\left|t \omega_{1} h_{1}^{\prime}+(1-t) \omega_{2} h_{2}^{\prime}\right|^{2} \\
= & t^{2}\left|h_{1}^{\prime}\right|^{2}\left(k^{2}-\left|\omega_{1}\right|^{2}\right)+(1-t)^{2}\left|h_{2}^{\prime}\right|^{2}\left(k^{2}-\left|\omega_{2}\right|^{2}\right) \\
& +2 t(1-t) \cdot \Re\left(\left(k^{2}-\omega_{1} \overline{\omega_{2}}\right) h_{1}^{\prime} \overline{h_{2}^{\prime}}\right) \\
\geq & 2 t(1-t) \cdot \Re\left(k^{2} h_{1}^{\prime} \overline{h_{2}^{\prime}}-g_{1}^{\prime} \overline{g_{2}^{\prime}}\right) \geq 0 .
\end{aligned}
$$

Hence $|\omega| \leq k<1$. Since $h_{1}-e^{2 i \gamma} g_{1}=\phi$ and $h_{2}-e^{2 i \gamma} g_{2}=\psi$, we have

$$
\begin{aligned}
h-e^{2 i \gamma} g & =\left[t h_{1}+(1-t) h_{2}\right]-e^{2 i \gamma}\left[\operatorname{tg}_{1}+(1-t) g_{2}\right] \\
& =t\left(h_{1}-e^{2 i \gamma} g_{1}\right)+(1-t)\left(h_{2}-e^{2 i \gamma} g_{2}\right)=t \phi+(1-t) \psi,
\end{aligned}
$$

which is convex in the direction $\gamma$ by the assumption. Thus, from Lemma 2, we know that $f \in \mathscr{S}_{H}(k)$ and is convex in the direction $\gamma$.

THeOREM 3. Let

$$
f_{1}=h_{1}+\overline{g_{1}} \in \mathscr{S}_{H}(k, \gamma ; \phi) \quad \text { and } \quad f_{2}=h_{2}+\overline{g_{2}} \in \mathscr{S}_{H}\left(k, \gamma+\frac{\pi}{2} ; \phi\right)
$$

where

$$
\phi(z)=\int_{0}^{z} \frac{e^{i \gamma} d \zeta}{\left(1+\zeta e^{i \theta}\right)\left(1+\zeta e^{-i \theta}\right)} \quad(\theta \in \mathbf{R})
$$


Suppose that

$$
\Re\left(k^{2} h_{1}^{\prime} \overline{h_{2}^{\prime}}-g_{1}^{\prime} \overline{g_{2}^{\prime}}\right) \geq 0,
$$

then $f=t f_{1}+(1-t) f_{2} \in \mathscr{S}_{H}(k)(0 \leq t \leq 1)$ and convex in the direction $\gamma$.

Proof. Making use of the similar arguments as in the proof of Theorem 2, in view of (9) and (10), we obtain that the dilatation $\omega$ of $f=t f_{1}+(1-t) f_{2}$ satisfies $|\omega| \leq k<1$.

Now we show that $f$ is convex in the direction $\gamma$. Note that

$$
h_{2}^{\prime}-e^{2 i \gamma} g_{2}^{\prime}=\left(h_{2}^{\prime}+e^{2 i \gamma} g_{2}^{\prime}\right)\left(\frac{h_{2}^{\prime}-e^{2 i \gamma} g_{2}^{\prime}}{h_{2}^{\prime}+e^{2 i \gamma} g_{2}^{\prime}}\right)=\phi^{\prime}(z)\left(\frac{1-e^{2 i \gamma} \omega_{2}}{1+e^{2 i \gamma} \omega_{2}}\right)=\phi^{\prime}(z) p(z),
$$

where

$$
p(z)=\frac{1-e^{2 i \gamma} \omega_{2}}{1+e^{2 i \gamma} \omega_{2}}
$$

satisfies $\Re(p(z))>0$. By setting $\kappa(z)$ by (3), we find that

$$
\begin{aligned}
\Re\left(\frac{z e^{-i \gamma}\left(h^{\prime}-e^{2 i \gamma} g^{\prime}\right)}{\kappa(z)}\right) & =\Re\left(\frac{z e^{-i \gamma}}{\kappa(z)}\left[t\left(h_{1}^{\prime}-e^{2 i \gamma} g_{1}^{\prime}\right)+(1-t)\left(h_{2}^{\prime}-e^{2 i \gamma} g_{2}^{\prime}\right)\right]\right) \\
& =t \cdot \Re\left(\frac{z e^{-i \gamma} \phi^{\prime}(z)}{\kappa(z)}\right)+(1-t) \cdot \Re\left(\frac{z e^{-i \gamma} \phi^{\prime}(z) p(z)}{\kappa(z)}\right) \\
& =t+(1-t) \Re(p(z))>0 .
\end{aligned}
$$

Therefore, by Lemma 3, we see that $e^{-i \gamma}\left(h-e^{2 i \gamma} g\right)$ is convex in the direction of the real axis, and hence the function $h-e^{2 i \gamma} g$ is convex in the direction $\gamma$. Furthermore, by Lemma 2 and Lemma 5, we conclude that $f \in \mathscr{S}_{H}(k)$, and it is convex in the direction $\gamma$.

Next, we prove the convexity of the linear combinations $f=t f_{1}+(1-t) f_{2}$ for the classes $\mathscr{S}_{H}^{-}(k ; \phi)$ and $\mathscr{S}_{H}^{+}(k ; \phi)$ for special $\phi$.

THEOREM 4. Let $f_{j}=h_{j}+\overline{g_{j}} \in \mathscr{S}_{H}^{-}(k ; \phi)(j=1,2)$, where

$$
\phi(z)=\frac{1}{2} \log \left(\frac{1+z}{1-z}\right) \quad(z \in \mathbf{D}) .
$$

Then $f=t f_{1}+(1-t) f_{2} \in \mathscr{S}_{H}^{-}(k ; \phi)(0 \leq t \leq 1)$, and $f(\mathbf{D})$ is convex.

Proof. In view of Corollary 4, we have $f=h+\bar{g} \in \mathscr{S}_{H}^{-}(k ; \phi)$, then by Lemma 2 the set $f(\mathbf{D})$ will be convex if and only if the analytic functions $h-e^{2 i \theta} g$ are univalent and convex in the direction $\theta$ for all $\theta, 0 \leq \theta<\pi$. To show the latter, it is sufficient to show that the functions $F_{\theta}=i e^{-i \theta}\left(h-e^{2 i \theta} g\right)$ are univalent and convex in the direction of the imaginary axis. 
Note that

$$
\begin{aligned}
h^{\prime}(z)-g^{\prime}(z) & =\left[t h_{1}^{\prime}(z)+(1-t) h_{2}^{\prime}(z)\right]-\left[\operatorname{tg}_{1}^{\prime}(z)+(1-t) g_{2}^{\prime}(z)\right] \\
& =t\left(h_{1}^{\prime}(z)-g_{1}^{\prime}(z)\right)+(1-t)\left(h_{2}^{\prime}(z)-g_{2}^{\prime}(z)\right) \\
& =\frac{1}{1-z^{2}} .
\end{aligned}
$$

Taking $\mu=v=\pi / 2$ in (4), we have

$$
\begin{aligned}
\Re\left(\left(1-z^{2}\right) F_{\theta}^{\prime}(z)\right) & =-\Im\left(e^{-i \theta}\left[h^{\prime}(z)-e^{2 i \theta} g^{\prime}(z)\right]\left(1-z^{2}\right)\right) \\
& =-\Im\left(\left[e^{-i \theta} h^{\prime}(z)-e^{i \theta} g^{\prime}(z)\right]\left(1-z^{2}\right)\right) \\
& =-\Im\left(\left[\left(h^{\prime}(z)-g^{\prime}(z)\right) \cos \theta-i\left(h^{\prime}(z)+g^{\prime}(z)\right) \sin \theta\right]\left(1-z^{2}\right)\right) \\
& =-\Im\left(\cos \theta-i \sin \theta \frac{h^{\prime}(z)+g^{\prime}(z)}{h^{\prime}(z)-g^{\prime}(z)}\right) \\
& =\Re(p(z)) \sin \theta \geq 0,
\end{aligned}
$$

where

$$
p(z)=\frac{h^{\prime}(z)+g^{\prime}(z)}{h^{\prime}(z)-g^{\prime}(z)}
$$

satisfies $\Re(p(z))>0$. Thus by Lemma 4 , we see that the function $F_{\theta}$ is univalent and convex in the direction of the imaginary axis.

In view of Theorem 4 and Lemma 6, we have the following result.

COROLlary 5. Let $f_{j}=h_{j}+\overline{g_{j}} \in \mathscr{S}_{H}^{-}(k ; \phi)(j=1,2, \ldots, n)$, where

$$
\phi(z)=\frac{1}{2} \log \left(\frac{1+z}{1-z}\right) \quad(z \in \mathbf{D}) .
$$

Then $f=\sum_{j=1}^{n} t_{j} f_{j} \in \mathscr{S}_{H}^{-}(k ; \phi) \quad\left(0 \leq t_{j} \leq 1, \sum_{j=1}^{n} t_{j}=1\right)$, and $f(\mathbf{D})$ is convex.

By similarly applying the method as in the proof of Theorem 4, we can easily get the following result for the class $\mathscr{S}_{H}^{+}(k ; \phi)$ for special $\phi$.

TheOREM 5. Let $f_{j}=h_{j}+\overline{g_{j}} \in \mathscr{S}_{H}^{+}(k ; \phi)(j=1,2)$, where

$$
\phi(z)=\frac{z}{1-z} \quad(z \in \mathbf{D}) .
$$

Then $f=t f_{1}+(1-t) f_{2} \in \mathscr{S}_{H}^{+}(k ; \phi) \quad(0 \leq t \leq 1)$, and $f(\mathbf{D})$ is convex.

Proof. By Corollary 3 with $A=1, B=0$ and $\alpha=-1$, we have $f=h+\bar{g} \in$ $\mathscr{S}_{H}^{+}(k ; \phi)$. In order to prove that $f(\mathbf{D})$ is convex, by Lemma 2 , it suffices to 
show that the analytic function $h-e^{2 i \theta} g$ is convex in the direction $\theta$ for every $\theta \in[0, \pi)$. The function $h-e^{2 i \theta} g$ is convex in the direction $\theta$ if and only if $F_{\theta}=i e^{-i \theta}\left(h-e^{2 i \theta} g\right)$ is convex in the direction of the imaginary axis.

Note that

$$
\begin{aligned}
h^{\prime}(z)+g^{\prime}(z) & =\left[t h_{1}^{\prime}(z)+(1-t) h_{2}^{\prime}(z)\right]+\left[t g_{1}^{\prime}(z)+(1-t) g_{2}^{\prime}(z)\right] \\
& =t\left(h_{1}^{\prime}(z)+g_{1}^{\prime}(z)\right)+(1-t)\left(h_{2}^{\prime}(z)+g_{2}^{\prime}(z)\right) \\
& =\frac{1}{(1-z)^{2}} .
\end{aligned}
$$

For $\theta \in[0, \pi / 2)$, taking $\mu=v=0$ in (4), we have

$$
\begin{aligned}
\Re\left(-i F_{\theta}^{\prime}(z)(1-z)^{2}\right) & =\Re\left(e^{-i \theta}\left[h^{\prime}(z)-e^{2 i \theta} g^{\prime}(z)\right](1-z)^{2}\right) \\
& =\Re\left(\left[e^{-i \theta} h^{\prime}(z)-e^{i \theta} g^{\prime}(z)\right](1-z)^{2}\right) \\
& =\Re\left(\left[\left(h^{\prime}(z)-g^{\prime}(z)\right) \cos \theta-i\left(h^{\prime}(z)+g^{\prime}(z)\right) \sin \theta\right](1-z)^{2}\right) \\
& =\Re\left(\frac{h^{\prime}(z)-g^{\prime}(z)}{h^{\prime}(z)+g^{\prime}(z)} \cos \theta-i \sin \theta\right) \\
& =\Re(p(z)) \cos \theta \geq 0,
\end{aligned}
$$

where

$$
p(z)=\frac{h^{\prime}(z)-g^{\prime}(z)}{h^{\prime}(z)+g^{\prime}(z)}
$$

satisfies $\Re(p(z))>0$. Therefore, by Lemma 4 , the function $F_{\theta}$ is convex in the direction of the imaginary axis for $\theta \in[0, \pi / 2)$. The same conclusion can be drawn for the function $F_{\theta}$ with $\theta \in[\pi / 2, \pi)$ if we apply Lemma 4 with $\mu=v=\pi$.

In view of Theorem 5 and Lemma 6, we have the following result.

CoROllary 6. Let $f_{j}=h_{j}+\overline{g_{j}} \in \mathscr{S}_{H}^{+}(k ; \phi)(j=1,2, \ldots, n)$ with

$$
\phi(z)=\frac{z}{1-z} \quad(z \in \mathbf{D}) .
$$

Then $f=\sum_{j=1}^{n} t_{j} f_{j} \in \mathscr{S}_{H}^{+}(k ; \phi) \quad\left(0 \leq t_{j} \leq 1, \sum_{j=1}^{n} t_{j}=1\right)$, and $f(\mathbf{D})$ is convex.

Acknowledgments. The authors would like to thank the referee for the helpful comments and suggestions. The research was supported by National Natural Science Foundation of China (Grant No. 11371126) and Academy of Finland and National Natural Science Foundation of China (Grant No. 269260). 


\section{REFERENCES}

[1] O. P. Ahuja, Use of theory of conformal mappings in harmonic univalent mappings with directional convexity, Bull. Malays. Math. Sci. Soc. (2) 35 (2012), 775-784.

[2] Z. Boyd, M. Dorff, M. NowaK, M. Romney AND M. Woloszkiewicz, Univalency of convolutions of harmonic mappings, Appl. Math. Comput. 234 (2014), 326-332.

[3] D. Bshouty, A. Lyzzaik and A. Weitsman, On the boundary behaviour of univalent harmonic mappings, Ann. Acad. Sci. Fenn. Math. 37 (2012), 135-147.

[4] D. M. Campbell, A survey of properties of the convex combination of univalent functions, Rocky Mount. J. Math. 5 (1975), 475-492.

[5] Sh. Chen, S. Ponnusamy and X. WAng, Integral means and coefficient estimates on planar harmonic mapping, Ann. Acad. Sci. Fenn. Math. 37 (2012), 69-79.

[6] J. Clunie and T. Sheil-Small, Harmonic univalent functions, Ann. Acad. Sci. Fenn. Ser. A. I. Math. 9 (1984), 3-25.

[7] M. Dorff, M. NowaK And M. Woloszkiewicz, Harmonic mappings onto parallel slit domains, Ann. Polon. Math. 101 (2011), 149-162.

[8] M. DorfF AND J. Rolf, Anamorphosis, mapping problems, and harmonic univalent functions, Explorations in complex analysis, Math. Assoc. of America, Inc, Washington, D.C., 2012, 197-269.

[9] P. Duren, Harmonic mappings in the plane, Cambridge University Press, Cambridge, 2004.

[10] W. Hengartner and G. Schober, Univalent harmonic functions, Trans. Amer. Math. Soc. 299 (1987), 1-31.

[11] X. Huang, Harmonic quasiconformal homeomorphism of the unit disk, Chinese Ann. Math. 29A (2008), 519-524.

[12] D. Kalaj and M. Pavlović, Boundary correspondence under harmonic diffeomorphisms of a half-plane, Ann. Acad. Sci. Fenn. Math. 30 (2005), 159-165.

[13] R. Kumar, S. Gupta and S. Singh, Linear combinations of univalent harmonic mappings convex in the direction of the imaginary axis, Bull. Malays. Math. Sci. Soc., DOI 10.1007/ s40840-015-0190-5.

[14] L. Li AND S. Ponnusamy, Injectivity of sections of univalent harmonic mappings, Nonlinear Anal. 89 (2013), 276-283.

[15] T. H. MacGRegor, The univalentce of a linear combination of convex mappings, J. London Math. Soc. 174 (1969), 210-212.

[16] S. Muir, Harmonic mappings convex in one or every direction, Comput. Methods Funct. Theory 12 (2012), 221-239.

[17] S. NagPal and V. Ravichandran, Univalence and convexiy in one direction of the convolution of harmonic mappings, Complex Var. Elliptic Equ. 59 (2014), 1328-1341.

[18] M. Pavlović, Boundary correspondence under harmonic quasiconformal homemorphisms of the unit disk, Ann. Acad. Sci. Fenn. Math. 27 (2002), 365-372.

[19] C. Pommerenke, On starlike and close-to-convex functions, Proc. London Math. Soc. 13 (1963), 290-304.

[20] W. C. Royster and M. Ziegler, Univalent functions convex in one direction, Publ. Math. Debrecen 23 (1976), 339-345.

[21] S. Y. Trimble, The convex sum of convex functions, Math. Z. 109 (1969), 112-114.

[22] Z. WANG, Z. LiU AND Y. Li, On the linear combinations of harmonic univalent mappings, J. Math. Anal. Appl. 400 (2013), 452-459.

[23] Z. WANG, Z. LiU AND Y. Li, On convolutions of harmonic univalent mappings convex in the direction of the real axis, J. Appl. Anal. Comput. 6 (2016), 145-155. 
[24] Z. WANG, L. Shi AND Y. JiAng, Construction of harmonic univalent mappings convex in one direction, Sci. Sin. Math. 44 (2014), 139-150 (in Chinese).

Yong Sun

SCHOOL OF MATHEMATICS AND ECONOMETRICS

HunAN UNIVERSITY

Changsha 410082, Hunan

P.R. CHINA

E-mail: yongsun2008@foxmail.com

Antti Rasila

Department of Mathematics and Systems Analysis

Aalto University

P. O. Box 11100, FI-00076 Aalto

FINLAND

E-mail: antti.rasila@iki.fi

Yue-Ping Jiang

SCHOOL OF MATHEMATICS AND ECONOMETRICS

HunAN UNIVERSITY

Changsha 410082, Hunan

P.R. CHINA

E-mail: ypjiang731@163.com 